\title{
Functional Characterization of CNGC19 and CNGC20 of Arabidopsis through CRISPR-Cas9
}

\author{
Tahreem Khalid ${ }^{1}$, Samia Hassan ${ }^{1}$, Sidra Ashraf $^{1}$, Hadia Naseem $^{1 *}$, Faiza Nasim ${ }^{1}$, Yasmeen Batool ${ }^{2}$ \\ ${ }^{1}$ Department of Biochemistry, University of Agriculture, Faisalabad, Pakistan \\ ${ }^{2}$ Department of Zoology, University of Agriculture, Faisalabad, Pakistan
}

\author{
DOI: $10.36348 / \mathrm{sijb} .2020 . \mathrm{v} 03 \mathrm{i} 06.004$
}

| Received: 15.06.2020 | Accepted: 22.06.2020 | Published: 26.06.2020

*Corresponding author: Hadia Naseem

\section{Abstract}

Arabidopsisthaliana belongs to family (Brassicaceae) of mustard plant. It is widely studied because it has short generation time with life cycle of 6 week. There 20 cyclic nucleotide gated channel $\left(\mathrm{CNGC}_{\mathrm{s}}\right)$ genes in Arabidopsisthalianaout of which CNGC19 and CNGC20 are mainly involved in abiotic stresses like drought and salinity. The concerted effort is made to identify the role of CNGCs especially CNGC20 in salinity. Mutant lines of CNGC20 have been activated also identified potential role CNGC19 and CNGC20 in salt stress tolerance through activation tagging. It is functionally characterized CNGC20 for their potential role in salt stress. Knock out (KO) line was obtained from Salk Center and homozygous KO line was not sensitive to salt stress. Over-expression lines were developed and similar to KO line OX lines were also not tolerant to salt stress compared with wild type. Moreover, there was no significant difference between fresh weight of root and shoot.In addition, it has also been confirmed through public microarray databases that CNGC19 and CNGC20 are activated under salt stress in Arabidopsis. So it designed CRISPR/Cas construct for CNGC20 to develop double mutant of CNGC19 and CNGC20. The results indicate that KO and OX lines of CNGC20 are not significantly different than wild type under salinity, when compared with wild-type. However interaction of both CNGC19 and CNGC20 may result in clear information about their role in salt stress.

Keywords: CRISPR Cas9, CNGC19, CNGC20, Arabidopsis thaliana, Knock out, Genome editing.

Copyright @ 2020: This is an open-access article distributed under the terms of the Creative Commons Attribution license which permits unrestricted use, distribution, and reproduction in any medium for non-commercial use (NonCommercial, or CC-BY-NC) provided the original author and source are credited.

\section{INTRODUCTION}

Arabidopsis thaliana is a non-woody stem plant described by Johannes Thal in $16^{\text {th }}$ century in Germany. This is also present in Central Asia, Europe, Northwest Africa and many other places as well. This plant is dicotyledonous; member of mustard family (Brassicaceaeorcruciferae) contains cabbage and radish species. From development to maturity its life cycle is about 6 weeks [1]. The first study of genome of this plant was done in 1996 and this genome was totally ordered after Drosophilla melanogaster and Caenorhabditis elegans [2]. This was third multicellular living thing and first plant whose genome has been identified. The flowers of plants have $0.5 \mathrm{~mm}$ developed seeds and $2 \mathrm{~mm}$ long in size. The central loop of plants has 4 white petals, 6 stamens and gynoecium and upper whorl of plant contained 4 green sepals. Additionally, this plant is related to about eleven thousand gene families and sequence of genome comparable to protein encoding genes, size of nuclear genome is $125 \mathrm{Mb}$ [3]. It explains a brief story of sequence of chromosomes with observed evolutionary history. The genomic study of this plant observed that it has individual genes [4].

Arabidopsis thaliana have twenty CNGCs which are classified into 4 groups (I-IV). Group II and group IV further divided into subdivisions (IIA and IIB, IVA and IVB). Group I contain gene CNGCs from monocotyledonous and dicotyledonous angiosperm plant. And group II gain CNGCs from soil plants and after the segregation from gydrophtic plants their ancestors are similar. Group II contains from lower soil plants like lycophytes and mosses. And Group IIA and III found CNGCs from embryophytes. And the last group IV contains it from vascular plants like embryophytes [5].

Abiotic tensions stimulate the plant structure and functions. Various changings have been observed because these stresses occur. Plants take up various changings, because of microorganisms reactions destroy by abiotic stresses which are stimulated by energetic signals. Due to abiotic strains various 
biochemical and physical responses are changed. Numerous genomic material function on transcriptional level to abiotic stresses [6]. The mechanism (genomic content transformation) stress supported genes have been observed into plants for raise their stress ability [7]. Abiotic tension tolerance holds various hundred genes. Osmotic pressure avoids the growth of plants with the change of active signaling which change cell production and functions [8]. Functions of various cations move on members and channels protein like glutamate receptor homologs and cyclic nucleotidegated ion channel have been in tension for determined biotic and abiotic tolerance. Growth of plant is controlled by CNGCs member who are determined for concerned in taking up $\mathrm{Na}^{+}, \mathrm{K}^{+}$and $\mathrm{Ca}^{+}$. Beside of biotic and abiotic task, CNGCs proteins defend pathways which are connected to growth and cellular ion channel homeostasis by work as guard cells [9].

CNGC19 and CNGC20 genes individually present in roots and branches of plants. At initial growth period, the CNGC19 found in roots and CNGC20 found in roots at early growth stage and CNGC20 shows more developmental functions in mesophyll cells nearby veins. Arrival of CNGC20 gradually increases in production and touch to saturation in growth and senescing leaves. Both genes activated in shoots due to response of increase in sodium chloride contents. Because of high salt construct existence of CNGC19 was completely absent in roots while it is present in shoots with specific activity examined in 6-72hrs. Similar to CNGC19, salty appearance of CNGC20 was also identified in shoots [10].

CRISPR-Cas9 certain nucleases produced as a particle of prokaryotic defense against viruses and commonly organized as tools to impose operatorspecified nucleotide arrangement stimulate in gene of interest [11]. This naturally mechanism used by ninety percent of archaea and forty percent bacteria as adaptive tolerance against violent virus or plasmid DNA. These useful techniques redesign the genetic engineering soil in fields [12]. CRISPR-Cas9 must be function in 1 time period of short plants because it is mostly agreeable to plant adaptations. By transgenic appearance this mechanism mainly show mosaic in $1^{\text {st }}$ generation (T1), expressing that technique-induced stimulate in this specie occurring after $1^{\text {st }}$ embryonic cell partition [13]. It might be present in animals and plants cells and they are easily penetrable to calcium ions. Calcium ions use these channels for responses; take as poisoned and vital cations and pathogenic defense in plant [14]. It is permeable in both monovalent and divalent cations by changes of cyclic nucleotide action. They act like calcium penetrable channels and they act on the site where calcium ions done their duty and some other pathways (like cyclic nucleotide pathway) act as protective procedure of plants. Two newly mutants of CNGCs of A. thaliana have been present and they stimulate response of microorganisms (15).

Drought and salinity period disturb the osmotic and ionic stability, stimulate the redox balance, cellular energy and damage the photosynthesis. Due of changing in development and growth of plant, critical loss of field production will be happened [16].

It is use in modern genomics by production of knock out (KO), various changings or stimulate action through sgRNA appearance for determined the response of activity of abiotic tolerance plants [17]. In future it became a best technique for specific gene editing in plant biology. Genomic redesigning of plant which have been presented recently, are best to commonly technique for development. It creates plants which are known as non-GM crops which are very unusual characteristics of the system. It may be better effect on breeding sector and plant biotechnology [18]. It develops a good tool for research in future, so mostly of them are in method of discovering. Many expectations in the case of agriculture to found the useful crops production with the help of this procedure for developed crops and use various biotech methods [19].

It is accurate engineering system which is depending on RNA-guided Cas9 nuclease from the type II prokarayotes CRISPR/Cas9 mechanism [20]. Locus of CRISPR was discovered in 1987 in bacteria (E. Coli) [21], on the other way protein Cas have been found in various prokaryotes [22]. CRISPR-Cas9 is an immune response adaptive by microbes that produce RNAguided nuclease to destroy the external DNA [23].

\section{REVIEW OF LITERATURE}

The plants that are easily attacked by pathogens are at risk to occur a specific disorder. First of all their immune system defended against pathogens [24]. However microorganisms release effector proteins to compete against immune system showing their resistance. Second way of defense occurs by genes that started protecting against pathogens of outsides. Products of $\mathrm{R}$ gene collaborate with the help of a virulent factor from microorganisms. $\mathrm{R}$ genes facilitated resistance release hypersensitive response (HR) on entry of pathogens [25]. After the introduction of pathogens, defended system is started to change the formation of reactive oxygen species (ROS) [26]. When infection of pathogens is started, calcium level is raised. Calcium act like second messenger and act in diversity of protective mechanism. One of them is product of ROS [27]. CNGCs taking as calcium carrying channels showing their action in immune system [28].

\section{Cyclic Nucleotide-gated channels (CNGCs)}

Cyclic nucleotide-gated channel (CNGC) participants represent a group of un-selective cation networks and allow $\mathrm{Na}+\mathrm{K}+$ and $\mathrm{Ca} 2+$ to be used [28]. It is assumed that CNG channels activate the ligand- 
binding site when binding cellular cAMP or cGMP. A partially overlap attached domain for calmodulin permit bind of calcium -calmodulin inside the channel's $\mathrm{C}$ terminus and is suggested to disrupt the open region. Workable appearance of plant CNGCs in Xenopus oocytes or animal cells has not yet been positively reproduced; therefore, a detailed biophysical description of these channels, such as the gating and permeation features remains to also be conducted. The CNGC gene family of Arabidopsis consists of 20 members [29].

\section{Physiological roles of $\mathrm{CNGC}_{\mathrm{s}}$ in plants}

cAMP and/or cGMP played a part in stomata opening, a short-term process involving multiple ion fluxes in crops. In Commelina, Auxin led stomata to be opened by high concentrations of cGMP and $\mathrm{Ca}_{2}{ }^{+}(30)$. $\mathrm{K}^{+}$channels in crops involving cAMP-dependent phosphorylation but direct binding of cyclic nucleotides to these channels would only lead in their activity being modified rather than gating. During stomatal opening or closing, cyclic nucleotide signals and $\mathrm{Ca}_{2}{ }^{+}$and $\mathrm{K}^{+}$ fluxes result in guar physiology.

CNGC plants played a significant role in taking cations non-selectively. While they work, pathogen acts in shoots. During signaling occurrences, CNGCs played a significant role in cross talking between cyclic nucleotides and $\mathrm{Ca}_{2}{ }^{+}$[14].

\section{Monovalent ion transportation by $\mathrm{CNGC}_{\mathrm{s}}$ \\ CNGCs include big gene families of} Arabidopsis involved in the transportation of monovalent ions (14). CNGC10 in crops was designated in $\mathrm{K}^{+}$uptake in prior research [31]. CNGC3 is also engaged in transportation of $\mathrm{Na}^{+}$and $\mathrm{K}^{+}$. Any disruption in CNGC3's function led in the content and structure of ions altering. In controlled circumstances, the development of mutant and wild crops was contrasted. On the other side, growth has been enhanced in the presence of surplus $\mathrm{Na}^{+}$in mutant crops compared to wild crops, in the existence of elevated $\mathrm{K}^{+}$ development in Arabidopsis has been reduced. Both ionselevated levels were fatal to crops. Growth was maintained by lowering the influx of ions, compartmentalizing elevated vacuoles. Ultimately, it was found that net $\mathrm{Na}^{+}$and $\mathrm{K}^{+}$uptake in mutant lines was reduced owing to variation in features of $\mathrm{CNGC} 3$ [32].

\section{Preparation of CRISPR-Cas construct for editing of Arabidopsis plants}

The sgRNA vectors along with the chosen U3 and U6 promoters were mixed with BsaIas well as linked to double stranded marked adaptors to produces gRNA characterization cassettes having marked arrangements sgRNA. Nested PCRs enhanced embedded sgRNA expression cassettes; secondary certain place PCRused readers, which contain primers for Golden Gate ligation with various BsaI-cutting locations [33].
The Golden Gate Ligation utilizes unique cutts function of the Type Irrestraint endonucleases, such as BsaI, to design and produce separate, nonalindromic sti ky ends of sequences that can prevent self-reliance linkage. However that technique was effective in connecting various DNA fragments in a single response in a designed order. Other cassette of site specific readers were also intended for in the vitro isothermal compatibility, called as Gibson Assembly [34], that have strong ability for connected to various genome particles with overlapping terminals. Furthermore, four pYLCRISPR/Cas9P35SH-depend construct having 1-3 AtU3 and/or AtU6 promoters-driven sgRNA expression cassettes were also ready to target genes in Arabidopsis. Through agrobacterium-mediated conversion we moved these 20 buildings into rice cultivars [35]. We have converted A. thaliana with 4 construct by using Agrobacterium technique to assess, however that scheme could be efficiently stimulate genes locations in the dicots plants [36].

\section{Targeted editing characterization in the transgenic Arabidopsis plants}

Evaluated targeted editing of crops obtained from the four constructs inA. Thaliana $\mathrm{T} 1 \quad\left(1^{\text {st }}\right.$ transgenic generation) to target six locations in four genes. We noted a range of targeted mutation kinds, including the addition of 1 or 2 nucleotides as well as removal of various nucleotide members. In 245 mutated locations analyzed (total of 490 alleles, not including similar wild typeand fragmentary excretion inside the 2 closely selectedlocations), large amountof the changed genetic material occurrence was single depended addition, most of that injected on $\mathrm{A}$ or $\mathrm{T}$ nucleotide in accordance with a prior study [37].

\section{Targeted editing of transgenic Arabidopsis crops}

The accurate editing of the candidate genes for their functional characterization is one of the main crop improvement demands. Newly findings of CRISPR/Cas9 bacterial scheme has provided marvelous potential for the meeting this requirement. Custam of the CRISPR/Cas9 was also expanded to include transcriptional regulation [38], epigenetic changings [39].

\section{CRISPR-Cas9 system variants}

The capacity of CRISPR/Cas9 to inform the accurate genome changes has had several latest success stories [13]. In order to improve its effectiveness, it is being further enhanced. Off-target genetic material changings are some of inevitable problems connected with this technology. sgRNA sometimes acknowledges nearly comparable un-target sequences inside the cell, with little base discrepancies leading to unnecessary, off-target mutations. Cas9 mutant for only one efficient domain (HNH or RuvC) is being used to decrease offtarget genetic changes with two sgRNAs having near target locations in reverse strands. Unlike Cas9, only at the target site, it is mutated version presents nick in one 
strand. Using this genetically altered Cas9 (nickase) with two sgRNAs having near target site causes close nicks in reverse strands resulting in a double strand tear that can be restored via NHEJ due to the target location mutation. However, owing to the lack of another near nick, DSB does not happen in the event of off-target nick. Strong-fidelity base excision repair path repairs off-target nickname [40]. Cas9's many version is dCas9, i.e. Cas9 lacking in nuclease. With the help of sgRNA, CRISPR-dCas9 can specifically bind to any sequence but cannot enter any genetic break. Different transcribing repressors or activators are merged to the CRISPR-dCas9 system respectively to alter target gene regulation or to hinder / enhance transcription [41].

\section{Salinity response for $\mathrm{CNGC}_{\mathrm{s}}$ expression}

CNGC19 and CNGC20 have been put in CNGC subgroup IVA and have also been present in tandem on chromosome 3. These two genes were identical in 73 percent. Both genes reacted under salinity to a signal of greater expression. Salinity has become a significant problem for crop productivity in Agriculture. To tackle this, under the condition of Saline, some processes became known to agricultural researchers [42]. The constant exposure of crops to greater salt concentration in shoots was the reason for greater $\mathrm{NaCl}$ but avoided development of the leaf. Some methods were adjusted by the crops to resist salinity, along with $\mathrm{Na}^{+}$suppression to reach roots, transportation and dispersal inside the leaf and extraction into vacuoles. Different shooting cells included separate ion concentrations such as barley and tomato with distinct $\mathrm{K}^{+}$and $\mathrm{Cl}^{+}$concentrations [43].

$\mathrm{Na}^{+}$was maintained in older leaves in Medicago citrine and Ricinuscommunis but avoided transportation to freshly established mature organs. It was moved to petioles and leaf margins from there in order that provide shield against increased $\mathrm{Na}^{+}$ production (44). Transporters played their roles in $\mathrm{Na}^{+}$ balancing in Arabidopsis, including plasma membrane (SOS1) and antiporters vacuolar $\mathrm{Na}^{+} / \mathrm{H}^{+}$(e.g. NHX1) and plasma membrane uniporter HKT1. By adding to the spreading of $\mathrm{Na}^{+}$within plant, CNGC19and CNGC20 enabled plant to deal with toxic impacts under salinity. Both genes under salinity were upregulated within hours.

CNGCs are engaged in controlling procedures of development and responding to abiotic and biotic tension. In reaction to developmental indications and salt stress, GFP, luciferase reporter assays and GUS are used for examine of expression of CNGC19and CNGC20genes from Arabidopsis thaliana. During early growth, major expression of CNGC19 was seen primarily in roots, while CNGC20 was shown in mesophyll cells round the veins. Both CNGC19 and CNGC 20 were displayed primarily in cultivated leaves. Both genes upregulation was seen in reaction to greater $\mathrm{NaCl}$ levels in Arabidopsisthaliana shoots. CNGC19 upregulation was not seen in reaction to greater roots level, but in shoots it was extremely up-regulated. In reaction to $75 \mathrm{mMNaCl}$ as in the wild type, similar TDNA addition lines of both genes were demonstrated phenotypically [45]. CNGCs hybrid with loss of sensation also facilitated their function in plant growth and pathogenic attack. For example, cngc3mutant seedlings showed mildly increased development relative to wild-type crops in the presence of greater $\mathrm{NaCl}$ or $\mathrm{KCl}$ levels [32]. Also tolerated for salt stress was CNGC10 [46].

\section{Abiotic Stressors}

Soil salinity has affected 80 million hectares (ha) of advanced land [47]. Based on a variety of biochemical and physiological modifications that further relied on gene expression, the crops were adjusted and tolerated to adverse circumstances [48]. These secondary messengers transmitted the stimulus and eventually engaged in stress-related expression of genes. Many stresses resulted in the cytosol's $\mathrm{Ca}_{2}{ }^{+}$ accumulation, which played a significant role as a secondary messenger in stimulating physiological mechanisms and gene expression that ultimately lead to stress response [49]. Plants were more prone to salinity harm when cellular $\mathrm{Ca}_{2}^{+}$was small [50]. High saline circumstances raised the $\mathrm{Ca}_{2}^{+}$-dependent sensing pathway that also meant that $\mathrm{Ca}_{2}{ }^{+}$conducting membrane proteins were strongly linked to plant salt tolerance. $\mathrm{CaM}$ managed to play their part in cooperation with $\mathrm{Ca}_{2}{ }^{+}$and decoding components in plant answer to salt strain [51].

Researchers also researched that the calcium signal transduction network in Arabidopsis is linked to the activation of the overly delicate salt (SOS) signaling network, which was essential to inregulate $\mathrm{Na}^{+}$and $\mathrm{K}^{+}$ homeostasis [52].

\section{Transcriptional Factors and stress Tolerance}

The transcription factor MYB96 acts a significant role in plant safety under pathogen infection in Arabidopsis by facilitating the molecular connection between both drought-induced ABA and pathogeninfected SA [53]. PR genes are also caused when a plant is exposed to abiotic pressure that ensures resistance to disease. The up-regulation of certain transcription factors following introduction to abiotic stress tends to the aggregation of PR proteins [53].

\section{Crisper/Cas system}

Crisper (Cluster of regularly interspersed brief palindromic sequence) model offers immunity to foreign invaders nucleic acid, along with viruses, used in bacteria and archaea as an innate and antiviral immune mechanism [54].

\section{Crisper concerned Discoveries}

Crisper also developed 48 percent in the sequence of bacterial genomes and 95 percent in 
Archeae's genetic sequence [55]. Archeae also recognized Crisper roles in 1993. First recognition of Cas genes was made in 2002 [55].

Crisper system functions in bacteria as a defensive mechanism in 2005 [22]. Crisper model included bacteriophageal immunity obtained in 2007 [54]. Crisper RNA's function in Crisper interference was developed in 2008 [56] as well as the role of spacers in phage mediated resistance [57]. The mechanism of crisper interference was recorded in 2010 [20]. Trans-activating crisper RNA (crRNA) was shown in 2011 [58]. Crisper / Cas instruments have been used in 2013 for effective mammalian gene editing instruments [59] as well as for effective mice genome engineering [60].

\section{Seeds Growth}

After seven days of incubation in the growing chamber (continuous fluorescent light, $20^{\circ} \mathrm{C}$ ) seedlings will be transferred into plastic pots having standard germination nutrients soil at $25{ }^{\circ} \mathrm{C}$ with specific light intensity given specifications. After transplanting of 3 weeks, plant attains finest developmental stage to be used for next procedure.

\section{Mechanism of action}

It consists of three stages.

\section{Integration}

The nucleic acid of the invader is incorporated into the host genome as a fresh spacer.

\section{Transcription}

Crisper is transcribed into early crisper RNA (pre-crRNA) and lastly mature crRNA is created through the cleavage method by endoribonuclease. The connection in both mature protein complex crRNA and Cas exists.

\section{Neutralization}

Finally, crRNA provides information to cas protein in order to neutralize the invader through the method of cleavage, resulting in virus inhibition and host tolerance.

\section{Spacers}

Some spacers suit viral genomes and plasmids [22]. Spacer sequences have been altered through genetic engineering, demonstrating that the existence of spacer gives opposition to phage while its lack constitutes a loss of opposition to phage [54]. Due to the similarity of spacers for both coding as well as noncoding viral DNA, Crisper / Cas process cuts dsDNA as goal.

\section{Leader}

The crisper leader comprises of less complicated, A/T wealthy non-coding sequences discovered in the upstream area performing role as a promoter for the transcription of early crisper RNA and speared to tiny RNA molecules that are connected to spacers placed in both two temporary repeats [61].

\section{Repeats}

Repeat units are situated within the locus of Crisper. There is a big amount of repeat events in microorganisms inside the crisper locus. Repeat sequence sizes range from 23 to $47 \mathrm{bp}$ [62].

\section{CONCLUSION}

Salinity and dryness pose the major threat to agricultural manufacturing and there has been a shortage of techniques that simultaneously interrupt various genes. CRISPR/Cas9 system is also useful and new technology for transgenic plant manufacturing. This new technology utilizes sgRNA to recognize targets. This scheme comprises of Cas endonuclease and sgRNA to recruit Cas9 as an additional target sequence double stranded break manufacturing. Transgenic plants are generated by means of the CRISPR / Cas scheme and assessed under drought and salinity. This novel technology also produced the double mutation of CNGCs in crops. The use of distinct markers can make over-expression chains of CNGCs.

\section{REFERENCES}

1. Magnus, N., \& Weigel, D. (2008). Next-generation in plants. Nat., 456: 720-723.

2. Adam, M. D., Cleniker, S. E., Holt, R. A., Evans, C. A., J. D Gocayne, P. G. (2000). Amanatides and R. A. George. 2000. The genome sequence of drosophilamelanogaster. Sci., 287: 2185-2195.

3. Carneiro, J. M. T., Madrid, K. C., Maciel, B. C. M., \& Arruda, M. A. Z. (2015). Arabidopsis thaliana and omics approaches. J. Intgr OMICS., 5: 1-16.

4. Kaul, S., Koo, H. L., Jenkins, J., Rizzo, M., Rooney, T., Tallon, L. J., \& Town C. D. (2000). Analysis of the genome sequence of the flowering plant Arabidopsis thaliana. Nat., 408: 796-815.

5. Li, W., Liu, B., Yu, L., Feng, D., Wang, H., \& Wang, J. (2009). Phylogenetic analysis, structural evolution and functional divergence of the 12-oxophytodienoate acid reductases gene family in plants. BMC Evol. Biol, 9: 90.

6. Shinozaki, K., \& Yamaguchi, K. S. (2000). Molecular responses to dehydration and low emperature: differences and cross-talk between two stress signaling pathways. Cur. Opin. Plnt. Biol., 3: 217 223.

7. Bajaj, L., Takai, M., Ahuja, R., Tang, K., Bagrodia, R., \& Gerla, M. (1999). Glomosim: A scalable network simulation environment. UCLA Comp. Sci. Depart. Tech. Rep, 990027: 213-216.

8. Zhu, J. K. (2001). Plant salt tolerance. Trnds. Plnt. Sci., 6: 66-71.

9. Köhler, C., \& Neuhaus, G. (2000). Characterisation of calmodulin binding to cyclic nucleotide- gated ion channels from Arabidopsis thaliana. Febs Lett., 471: 133-136.

10. Borsics, T., Webb, D., Andeme, O. C., Staehelin, L. A., \& Christopher, D. A. (2007). The cyclic 
nucleotide-gated calmodulin-binding channel AtCNGC10 localizes to the plasma membrane and influences numerous growth responses and starch accumulation in Arabidopsis thaliana. Plnt., 225: 563573.

11. Jinek, M., Jiang, F., Taylor, D. W., Sternberg, S. H., Kaya, E., Ma, E., Anders, C., Hauer, M., Zhou, K., Lin, S., \& Kaplan, M. (2014). Structures of Cas9 endonucleases reveal RNA-mediated conformational activation. Sci., 343: 1247997-1247998.

12. Sorek, R., Kunin, V., \& Hugenholtz, P. (2008). CRISPR a widespread system that provides acquired resistance against phages in bacteria and archaea. Nat. Rev. Microb, 6: 181-186.

13. Feng, Z., Mao Y., N. Xu, B. Zhang, P. Wei, D. L. Yang, Z. Wang, Z. Zhang, R. Zheng, L. Yang and L. Zeng. (2014). Multigeneration analysis reveals the inheritance, specificity and patterns of CRISPR/Casinduced gene modifications in Arabidopsis. Proceed. Nat. Acad. Sci., 111: 4632-7.

14. Talke, I. N., Blaudez, D., Maathuis, F. J., \& Sanders, D. (2003). CNGCs: prime targets of plant cyclic nucleotide signalling? Trnds. Plnt. Sci., 8: 286-293.

15. Kugler, A., Kohler, B., Palme, K., x Wolff, K., \& Dietrich, P. (2009). Salt-dependent regulation of a CNG channel subfamily in Arabidopsis. BMC Plnt. Biol., 9: 1-5.Kugler, A., B. Köhler, K. Palme, P. Wolff and P. Dietrich. 2009. Salt-dependent regulation of a CNG channel subfamily in Arabidopsis. BMC Plant Biol., 9: 140.

16. Golldack, D., Li, C., Mohan, H., \& Probst, N. (2014). Tolerance to drought and salt stress in plants: unraveling the signaling networks. Fronti. Plnt. Sci., 5: $15-24$.

17. Jain, M. (2015). Function genomics of abiotic stress tolerance i.n plants: a CRISPR approach. Fronti. Plnt. Sci., 6: 375-379.

18. Belhaj, K., Chaparro, A. G., Kamoun, S., \& Nekrasov, V. (2013). Plant genome editing made easy: targeted mutagenesis in model and crop plants using the CRISPR/Cas system. Plnt Methd, 9: 39.

19. Lusser, M., \& Davies, H. V. (2013). Comparative regulatory approaches for groups of new plant breeding techniques. New Biotech, 30: 437-446.

20. Garneau, J. E., Dupuis, M. E., Villion, M., Romero, D. A., Barrangou, R., Boyaval, P., Fremaux, C., Horvath, P., Magadán, A. H., \& Moineau, S. (2010). The CRISPR/Cas bacterial immune system cleaves bacteriophage and plasmid DNA. Nat, 468: 67-71.

21. Ishino, Y., Shinagawa, H., Makino, K., Amemura, M., \& Nakata, A. (1987). Nucleotide sequence of the iap gene, responsible for alkaline phosphatase isozyme conversion in Escherichia coli, and identification of the gene product. Jr. Bacterial., 169: 5429-5433.

22. Mojica, F. J., Díez, V. C., Soria, E., \& Juez, G. (2000). Biological significance of a family of regularly spaced repeats in the genomes of Archaea, Bacteria and mitochondria. Mol. Microb, 36: 244246.

23. Bhaya, D., Davison, M., \& Barrangou, R. (2011). CRISPR-Cas systems in bacteria and archaea: versatile small RNAs for adaptive defense and regulation. Annu. Rev. Genet, 45: 273-297.
24. Zhang, J., \& Zhou, J. M. (2010). Plant immunity triggered by microbial molecular signatures. Mol. Plant., 3: 783-793.

25. Bent, A. F., \& Mackey, D. (2007). Elicitors, effectors and R gene: the new paradigmand a lifetime supply of questions. Annu. Rev. Phytopathol, 45: 399-436.

26. Torres, M. A. (2010). ROS in biotic interactions. PhysiolPlantarum, 138: 414-429.

27. Sasabe, M., Takeuchi, K., Kamoun, S., Ichinose, Y., Govers. F., Toyoda, K., Shiraishi, T., \& Yamada, T.(2000). Independent pathways leading to apoptotic cell death, oxidative burst and defense gene expression in response to elicitin in tobacco cellsuspension culture. Eur. J. Biochem, 267: 50055013.

28. Kaplan, B., Sherman, T., \& Fromm, H. (2007). Cyclic nucleotide-gated channels in plants.FEBS Lett, 581: 2237-2246.

29. Mäser, P., Thomine, S., Schroeder, J. I., Ward, J. M., Hirschi, K., Sze, H., ... \& Harper, J. F. (2001). Phylogenetic relationships within cation transporter families of Arabidopsis. Plant Physiology, 126(4), 1646-1667.

30. Cousson, A. (2001). Pharmacological evidence for the implication of both cyclic GMP-dependent andindependent transduction pathways within auxininduced stomatal opening in Commelina communis (L.). Plant Science, 161(2), 249-258.

31. Liu, Y. G., Chen, Y., \& Zhang, Q. (2005). Amplification of genomic sequences flanking T-DNA insertions by thermal asymmetric interlaced polymerase chain reaction. In Transgenic Plants: Methods and Protocols (pp. 341-348). Humana Press.

32. Gobert, A., Park, G., Amtmann, A., Sanders, D., \& Maathuis, F. J. (2006). Arabidopsis thaliana cyclic nucleotide gated channel 3 forms a non-selective ion transporter involved in germination and cation transport. Journal of experimental botany, 57(4), 791800.

33. Engler, C., Kandzia, R., \& Marillonnet, S. (2008). A one pot, one step, precision cloning method with high throughput capability. PloS one, 3(11).

34. Gibson, D. G., Young, L., Chuang, R. Y., Venter, J. C., Hutchison, C. A., \& Smith, H. O. (2009). Enzymatic assembly of DNA molecules up to several hundred kilobases. Nature methods, 6(5), 343-345.

35. Hiei, Y., Ohta, S., Komari, T., \& Kumashiro, T. (1994). Efficient transformation of rice (Oryza sativa L.) mediated by Agrobacterium and sequence analysis of the boundaries of the T- DNA. The Plant Journal, 6(2), 271-282.

36. Clough, S. J. and A. F. Bent 1998. Floral dip: a simplified method for Agrobacterium-mediated transformation of Arabidopsis thaliana. Plant J., 16:735-743.

37. Zhang, H., Zhu, H., Pan, Y., Yu, Y., Luan, S., \& Li, L. (2014). A DTX/MATE-type transporter facilitates abscisic acid efflux and modulates ABA sensitivity and drought tolerance in Arabidopsis. Molecular plant, 7(10), 1522-1532.

38. Cheng, A. W., Wang, H., Yang, H., Shi, L., Katz, Y., Theunissen, T. W., ... \& Jaenisch, R. (2013). Multiplexed activation of endogenous genes by 
CRISPR-on, an RNA-guided transcriptional activator system. Cell research, 23(10), 1163-1171.

39. Hu, J., Lei, Y., Wong, W. K., Liu, S., Lee, K. C., He, X., \& Peng, X. (2014). Direct activation of human and mouse Oct 4 genes using engineered TALE and Cas9 transcription factors. Nucleic acids research, 42(7), 4375-4390.

40. Ran, F. A., Hsu, P. D., Lin, C. Y., Gootenberg, J. S., Konermann, S., Trevino, A. E., ... \& Zhang, F. (2013). Double nicking by RNA-guided CRISPR Cas9 for enhanced genome editing specificity. Cell, 154(6), 1380-1389.

41. Gilbert, L. A., Horlbeck, M. A., Adamson, B., Villalta, J. E., Chen, Y., Whitehead, E. H., ... \& Qi, L. S. (2014). Genome-scale CRISPR-mediated control of gene repression and activation. Cell, 159(3), 647-661.

42. Munns, R., \& Tester, M. (2008). Mechanisms of salinity tolerance. Annu. Rev. Plant Biol., 59, 651681.

43. James, R. A., Munns, R., Von Caemmerer, S., Trejo, C., Miller, C., \& Condon, T. (2006). Photosynthetic capacity is related to the cellular and subcellular partitioning of $\mathrm{Na}+, \mathrm{K}+$ and $\mathrm{Cl}-$ in salt- affected barley and durum wheat. Plant, Cell \& Environment, 29(12), 2185-2197.

44. Sibole, J. V., Cabot, C., Poschenrieder, C., \& Barceló, J. (2003). Ion allocation in two different salt-tolerant MediterraneanMedicagospecies. Journal of plant physiology, 160(11), 1361-1365.

45. Kugler, A., Köhler, B., Palme, K., Wolff, P., \& Dietrich, P. (2009). Salt-dependent regulation of a CNG channel subfamily in Arabidopsis. BMC Plant Biology, 9(1), 140.

46. Guo, K. M., Babourina, O., Christopher, D. A., Borsics, T., \& Rengel, Z. (2008). The cyclic nucleotide- gated channel, AtCNGC10, influences salt tolerance in Arabidopsis. Physiologia Plantarum, 134(3), 499-507.

47. Zhang, H., Han, B., Wang, T., Chen, S., Li, H., Zhang, Y., \& Dai, S. (2012). Mechanisms of plant salt response: insights from proteomics. Journal of proteome research, 11(1), 49-67.

48. Bhatnagar-Mathur, P., Vadez, V., \& Sharma, K. K. (2008). Transgenic approaches for abiotic stress tolerance in plants: retrospect and prospects. Plant cell reports, 27(3), 411-424.

49. Luan, S. (2009). The CBL-CIPK network in plant calcium signaling. Trends in plant science, 14(1), 3742.

50. Hong-Bo, S., Zong-Suo, L., \& Ming-An, S. (2005). LEA proteins in higher plants: structure, function, gene expression and regulation. Colloids and surfaces B: Biointerfaces, 45(3-4), 131-135.
51. Hong-Bo, S., Li-Ye, C., Ming-An, S., Shi-Qing, L., \& Ji-Cheng, Y. (2008). Bioengineering plant resistance to abiotic stresses by the global calcium signal system. Biotechnology advances, 26(6), 503-510.

52. Jackson, H. A., Marshall, C. R., \& Accili, E. A. (2007). Evolution and structural diversification of hyperpolarization-activated cyclic nucleotide-gated channel genes. Physiological genomics, 29(3), 231245 .

53. Seo, P. J., Kim, M. J., Park, J. Y., Kim, S. Y., Jeon, J., Lee, Y. H., ... \& Park, C. M. (2010). Cold activation of a plasma membrane- tethered NAC transcription factor induces a pathogen resistance response in Arabidopsis. The Plant Journal, 61(4), 661-671.

54. Barrangou, R., Fremaux, C., Deveau, H., Richards, M., Boyaval, P., Moineau, S., \& Horvath, P. (2007). CRISPR provides acquired resistance against viruses in prokaryotes. Science, 315(5819), 1709-1712.

55. Jansen, R., Embden, J. D. V., Gaastra, W., \& Schouls, L. M. (2002). Identification of genes that is associated with DNA repeats in prokaryotes. Molecular microbiology, 43(6), 1565-1575.

56. Brouns, S. J., Jore, M. M., Lundgren, M., Westra, E. R., Slijkhuis, R. J., Snijders, A. P., ... \& Van Der Oost, J. (2008). Small CRISPR RNAs guide antiviral defense in prokaryotes. Science, 321(5891), 960-964.

57. Deveau, H., Barrangou, R., Garneau, J. E., Labonté, J., Fremaux, C., Boyaval, P., \& Moineau, S. (2008). Phage response to CRISPR-encoded resistance in Streptococcus thermophilus. Journal of bacteriology, 190(4), 1390-1400.

58. Deltcheva, E., Chylinski, K., Sharma, C. M. Gonzales, K., Chao, Y., Pirzada, Z. A., \& Charpentier, E. (2011). CRISPR RNA maturation by trans-encoded small RNA and host factor RNase III. Nature, 471(7340), 602-607.

59. Cong, L., Ran, F. A., Cox, D., Lin, S., Barretto, R., Habib, N., \& Zhang, F. (2013). Multiplex genome engineering using CRISPR/Cas systems. Science, 339(6121), 819-823.

60. Yang, H., Wang, H., Shivalila, C. S., Cheng, A. W., Shi, L., \& Jaenisch, R. (2013). One-step generation of mice carrying reporter and conditional alleles by CRISPR/Cas-mediated engineering. Cell, 154(6), 1370-1379.

61. Lillest $\varnothing 1$, R. K., Shah, S. A., Brügger, K., Redder, P., Phan, H., Christiansen, J., \& Garrett, R. A. (2009). CRISPR families of the crenarchaeal genus Sulfolobus: bidirectional transcription and dynamic properties. Molecular microbiology, 72(1), 259-272.

62. Grissa, I., Vergnaud, G., \& Pourcel, C. (2007). The CRISPRdb database and tools to display CRISPRs and to generate dictionaries of spacers and repeats. BMC bioinformatics, 8(1), 172. 